\title{
Design and Implementing of Serial Ports to Ethernet Gateway on Embedded System
}

\author{
R.V. Sonawane, A.A. Naik \\ Department of Electronics \& Tele Communication, Maharashtra Institute of Technology, Pune, India
}

\begin{abstract}
The use of the multi-serial/Ethernet conversion gateway simplifies the networked control and distributed management of information for serial devices. So the design of a new type of gateway for data converting from serial ports to Ethernet based on Embedded system is highlighted in this paper. The design is based on ARM7 (LPC2138) processor and serial ports to Ethernet data conversion gateway that will convert the serial data of various components to Ethernet data. The communication between the serial devices and monitor computer is simplified with the Ethernet protocol. Data transmission from number of serial devices used in industrial applications can be effectively achieved. The use of such system will improve the CPU efficiency of the system to ensure real-time data processing.
\end{abstract}

Keywords - Ethernet; gateway; ARM7; serial ports; TCP/IP protocol.

\section{Introduction}

There is a strong interest of using cheap and simple Ethernet technology for control networks. Its advantage of low price and robustness, resulting from its wide acceptance and deployment, has created an eagerness to meet the real time requirements.

Microcontrollers are low cost embedded systems that control and monitor no. of appliances. Enabling a microcontroller to a ubiquitous data communication network e.g the Ethernet network, will allow developers and end users to monitor and control microcontroller operated devices with greater flexibility.

The communications between existing computer monitor and equipment are all standard forms of asynchronous serial, the controller is connected with the various components by serial port expansion. The use of the process system makes frequent interruptions of the computer and greatly reduces the efficiency of the CPU. So a new type of system design based on Serial ports to Ethernet data conversion gateway, to convert the serial data of various components to Ethernet data, simplifying the communication between the various components and monitor computer only with the protocol of Ethernet.[1]

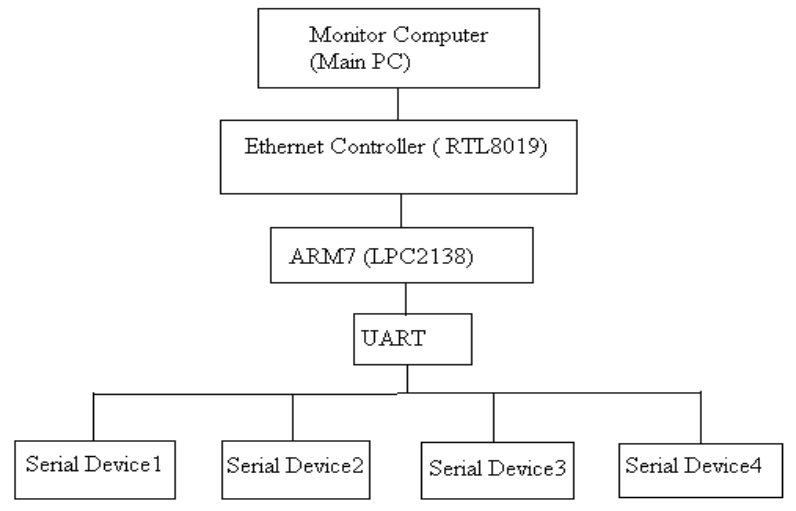

Figure 1. System Diagram

As seen in Fig. 1, the communication between the serial devices and computer takes place through the gateway. The gateway will send data from serial devices to the Computer and the control commands from the computer in the Ethernet format will be converted to serial frame format and then send to each serial device. [2]

\section{Methodology}

The entire system will be designed using ARM development board and Ethernet development board.

The functional diagram of the system is mainly divided into two main sections:

1) Hardware interface unit (RTL8019AS)

2) Processing unit (LPC2138) 
Hardware interface unit (RTL8019AS):

An embedded system that supports Ethernet requires Ethernet controller and a cable to provide network interface. The board consists of Ethernet controller. It is complaint to Ethernet II and IEEE 802.310 base5, 10 base2, 10 baseT Ethernet interface.

Processing unit (LPC2138):

In the Ethernet capable embedded system a CPU manages communication with the Ethernet controller. The minimum requirement for the CPU is a microcontroller with external 8 bit data bus.

The Ethernet controller hardware normally handles the sending and receiving of frames including detecting collision and deciding when to try again after collision. The CPU writes the data to send into memory that the CPU can access and the controller stored the received data in the memory that the CPU can access.

The system is based on high-speed Ethernet communication technique and Real-time Operating System (RTOS). It is a serial equipment which incorporates Ethernet with serial data transmission. [2]

The design is based on ARM7 (LPC2138) processor and serial ports to Ethernet data conversion gateway that converts the asynchronous serial data of various components to Ethernet data. ARM7 microprocessor (LPC2138) is used and its parallel port is expanded for communication using Quad UART (ST16C554) with serial ports data. RTL8019 is a highly integrated Ethernet Controller used for connecting the ARM board to the Ethernet port of the PC. The processor will take the data from the RTL8019 decode it and accordingly send it to the corresponding PC.[3]

The project consists of two primary elements

1) server consisting of ARM7 microcontroller, LPC2138, interfaced with Ethernet IC, Realtek RTL8019AS and

2) no. of serial devices connected to the system through Quad UART.

The serial devices send/ receive data to/ from the microcontroller using TCP packets. The transfer speed of Ethernet has developed to 10Gbps from initial 10Mbps.

\section{Hardware Design}

The hardware consists of ARM Processor (LPC2138), Ethernet Controller (RTL8019) and Quad UART (ST16C554).

The Ethernet controller hardware normally handles the sending and receiving of frames including detecting collision and deciding when to try again after collision. The CPU writes the data to send into memory that the CPU can access and the controller stored the received data in the memory that the CPU can access. [5] The LPC2138 microcontrollers are based on a 16/32-bit ARM7TDMI-S CPU with real-time emulation and embedded trace support, that combine the microcontroller upto $512 \mathrm{kB}$ of embedded high-speed flash memory. A 128-bit wide memory interface and a unique accelerator architecture enable 32-bit code execution at maximum clock rate. 16-bit Thumb mode reduces code by more than $30 \%$ with minimal performance penalty. Due to the low power consumption, these microcontrollers are ideal for applications where miniaturization is a key requirement.

Ethernet has become the most popular LAN scheme in last decade. It is simple, efficient, easily integration and bus based architecture is particularly used for wide applications. The system is based on highspeed Ethernet communication technique and Real-time Operating System (RTOS). It is a serial equipment which incorporates Ethernet with serial data transmission. [6]

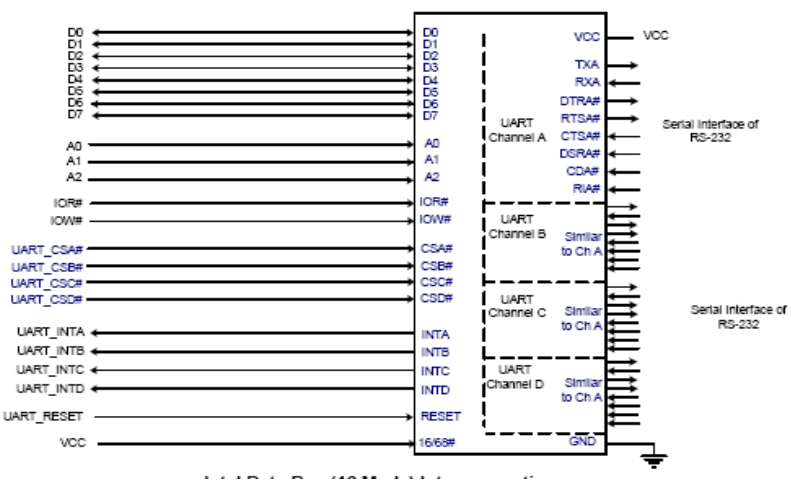

Figure 2: Interface of the Microcontroller with Quad UART

As seen in Fig. 2, all the four UART channels share the same bus for host operations. The interface is 8 data bits wide with 3 address lines and control signals to execute data bus read and write transactions.

Working of Ethernet controller 
Ethernet communications are typically handled by a combination of an Ethernet Controller chip and device driver code that communicates with the controller. The Ethernet controller handles the sending and receiving of Ethernet frames.

In sending a frame, a controller will do the following

1) Receives the message to send and destination address.

2) Calculates the Ethernet frame check sequence.

3) Places data, address and other information in the frames field.

4) Attempts to transmit the frame when the network is idle

5) Half duplex interface detects collisions, cancels any transmitted frame with a collision and tries according to protocol standard IEEE802.3; in full duplex Ethernet segment doesnot need to support collision detecting because there are no collisions to detect.

6) Provides an indication of success or failure of a transmission.

In receiving a frame, a controller will do the following

1) Detects and synchronizes to new received frames.

2) Ignores if any frames that is less than the minimum size.

3) Ignores if any frames that donot contain the interfaces address or a valid multicast or broadcast address in the Destination Address field.

4) Calculates the frame check sequence value, compares the result with the received value and indicates the errors if they dont match.

5) Makes the received frames data and other information available to the receiving computer and reads the data.

Description of Software

\section{Software Design}

It mainly realizes conversion processing between serial data and Ethernet data. It can identify a serial port through IP address and the NO. of port. It is developed using Keil software. It includes RTL8019AS control functions and all needed communications( ARP, IP, TCP). [2]

Frame format

\begin{tabular}{c|c|c|c}
\hline Synchronous head 1 & Synchronous head 2 & data \\
\hline \multicolumn{4}{c}{ Figure 3: Frame format of serial data } \\
\hline $\begin{array}{c}\text { Synchronous head } \\
1\end{array}$ & $\begin{array}{c}\text { Synchronous head } \\
2\end{array}$ & $\begin{array}{c}\text { Serial } \\
\text { Number }\end{array}$ & data \\
\hline
\end{tabular}

Figure 4: Frame format of Ethernet data

The received data of serial device must go through as part of Ethernet frames after the package, and Ethernet must analyse all receive data, in which way control data will be accurately sent to each serial equipment, which requires the serial data frame and Ethernet data frame for a unified treatment.Ethernet transmits data using UDP communication protocol through IP address aiming at high stability. [4] 


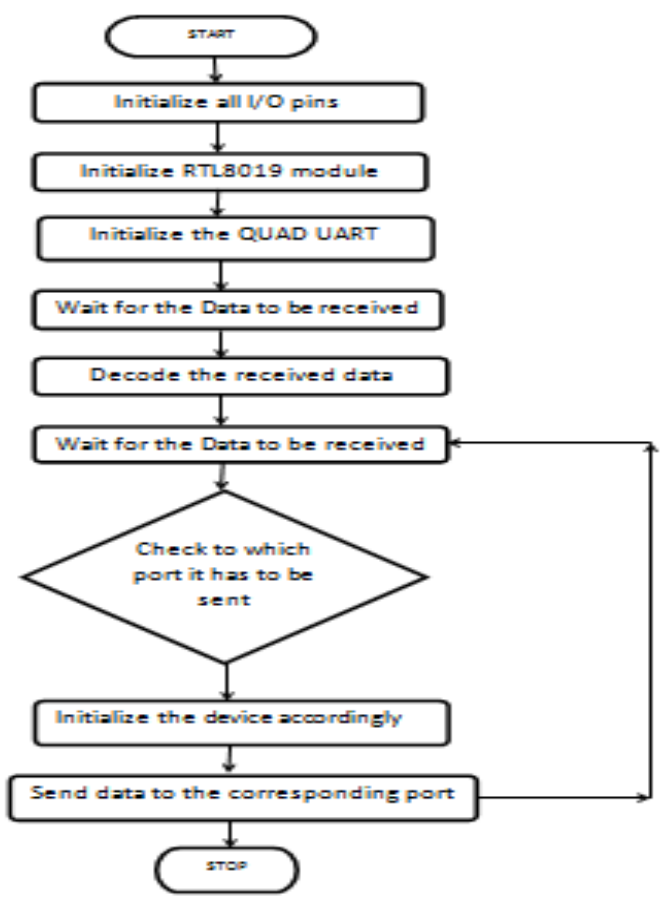

Figure 5: Flowchart of the system

\section{CONCLUSION}

The paper proposes embedded system architecture based on real-time Ethernet. It will provide a uniform environment for integration of real-time control and information exchange. Using Ethernet as the communications backbone for the embedded systems has several advantages. In recent years, Ethernet network protocol has been widely adopted as the choice method of data communication for personal computers and other digital devices. Its popularity is due to the immense use of the Internet, an information exchange infrastructure that communicates the data via the Ethernet network. Furthermore Ethernet is readily available on most of the currently deployed PCs. as a data communication protocol. Such systems can be used in electric power net automatic system, transmission and distribution of electric power control system, Internet/LAN based systems, long-distance video frequency transmission system, industry/factory's automatic system and SCADA system.

\section{References}

[1] Yonghong Hu; Lu Ding; Tong Yao; "Designing and Implementing of Serial Ports to Ethernet Gateway on Embedded System" Computer Network and Multimedia Technology, 2009. CNMT 2009. International Symposium, Beijing China.

[2] Lingbo Zhu, Guanzhong Dai, Li Shi, Xuefang Lin-shi, "Modeling and Implementation for embedded DC Motor Ethernet Control System", 2008 International Conference on Computer Science and Software Engineering., pp9-12.

[3] Jinghua Wu, Meng Zhang, Jun Ji. "Design of Embedded Serial Data-Network Converter," Computational Engineering in Systems Applications, 2006 The Proceedings of the Multiconference on; Beijing,China. pp.784-788.

[4] Li-wei Wu, Jwn-Sheng Hu. "Distributed Embedded Real-Time Ethernet Platform for Robots Control" Proceeding of the 2005 IEEE international Conference on Mechatronics ,July 10-12,2005,Taipei,TaiWan. pp.370- 375.

[5] Dal-Hwan Yoon; Ye Heon Jung; Hak Jong Ryoo; Hyung Mook Kim; Sung Yong Choei "The Gateway Implementation of Sensor Network using the ARM Chip," Advanced Communication Technology, 2006. ICACT 2006. The 8th International Conference Volume 1, Issue , 20-22 Feb. 2006 , Phoenix park, Korea pp.146-148.

[6] Yanjun FANG, Bo XI Meicheng CHEN \& Jingyu LIU, "Implementation of Industrial Ethernet Commumication Based on Embedded Systems" International Conference 2006 IEEE.

[7] Online: http://www.semiconductors.philips.com for ARM7 LPC2138 Datasheet, User manual.

[8] Online: http://www.realtek.com.tw for RTL8019 Datasheet.

[9] Online: http://in.element14.com for QUAD UART Datasheet. 